\title{
NEW RESULTS ON THE POMPEIU PROBLEM
}

\author{
NICOLA GAROFALO AND FAUSTO SEGALA
}

\begin{abstract}
Let $p_{N}(w)=\sum_{k=0}^{N} a_{k} w^{k}, w \in \mathbb{C}, N \in \mathbb{N}$, be a polynomial with complex coefficients. In this paper we prove that if $D \subset \mathbb{R}^{2}$ is a simplyconnected bounded open set whose boundary is a closed, simple curve parametrized by $x(s)=x_{1}(s)+i x_{2}(s)=p_{N}\left(e^{i s}\right), s \in[-\pi, \pi]$, then $D$ has the Pompeiu property unless $N=1$ and $p_{1}(w)=a_{1} w+a_{2}$ in which case $D$ is a disk. This result supports the conjecture that modulo sets of zero twodimensional Lebesgue measure, the disk is the only simply-connected, bounded open set which fails to have the Pompeiu property.
\end{abstract}

\section{INTRODUCTION}

Formulated by the Roumanian mathematician D. Pompeiu [P1, P2] in 1929, the Pompeiu problem consists in characterizing those bounded sets $D \subset \mathbf{R}^{2}$ for which $f \equiv 0$ is the only continuous function on $\mathbf{R}^{2}$ such that

$$
\int_{\sigma(D)} f(x) d x=0, \quad \text { for every rigid motion } \sigma \text { of } \mathbf{R}^{2} .
$$

A set $D \subset \mathbf{R}^{2}$ for which $f \equiv 0$ is the only function such that (1.1) holds is said to have the Pompeiu property. Although this was not realized by Pompeiu himself, invariance with respect to rotations causes failure of the Pompeiu property. For instance, disks or annuli do not have the Pompeiu property (see [C]), whereas elliptical regions do (see [BST]). It is very tempting to conjecture that: Modulo sets of two-dimensional Lebesgue measure zero, the disk is the only bounded, simply connected open subset of $\mathbf{R}^{2}$ that does not have the Pompeiu property.

In 1973 Brown, Schreiber, and Taylor [BST] proved that every polygonal region, or, more generally, every convex set with at least a true corner has the Pompeiu property. In 1976 Williams [W1] proved a remarkable connection between the Pompeiu problem and a symmetry problem in partial differential equations, known as Schiffer's conjecture:

Received by the editors November 18, 1988 and, in revised form, April 24, 1989.

1980 Mathematics Subject Classification (1985 Revision). Primary 30E15, 41A60; Secondary $42 \mathrm{~A} 38$.

The first author was supported by the National Science Foundation, grant DMS-8905338. 
Let $D \subset \mathbf{R}^{2}$ be a bounded, connected open set with $C^{2}$ boundary. Does the existence of a nontrivial solution $u$ of the overdetermined eigenvalue problem

$$
\left\{\begin{array}{l}
\Delta u=-\lambda u \text { in } D, \quad \lambda>0, \\
\left.u\right|_{\partial D}=\text { const. },\left.\quad \frac{\partial u}{\partial \nu}\right|_{\partial D}=0,
\end{array}\right.
$$

imply that $D$ is a ball?

It was proved in [W1] that for a domain $D \subset \mathbf{R}^{2}$ failure of the Pompeiu property is equivalent to the existence of a nontrivial solution of (1.2). In 1981 Williams [W2], following the approach in [Ca], proved a free boundary result concerning (1.2). As a consequence of it in $\mathbf{R}^{2}$ one obtains

Theorem A. Let $D \subset \mathbf{R}^{2}$ be a bounded, simply-connected open set whose boundary $\partial D$ is Lipschitz. If $\partial D$ is not real analytic, $D$ has the Pompeiu property.

The results in [BST] and [W2] leave open the case of domains with analytic boundaries. In 1982 Brown and Kahane [BK] proved the following

Theorem B. Let $D \subset \mathbf{R}^{2}$ be a bounded, convex set with real analytic boundary. Let $m(D)$ and $M(D)$ respectively denote the minimum and maximum diameter of $D$. If

$$
m(D) \leq \frac{1}{2} M(D),
$$

then $D$ has the Pompeiu property.

In this paper we give a contribution to the above conjecture. We single out a class of domains in $\mathbf{R}^{2}$ that have the Pompeiu property. Our main result is the following

Theorem. Let $D \subset \mathbf{R}^{2}$ be a bounded, simply-connected open set whose boundary $\partial D$ is a closed, simple curve parametrized by $x(s)=\left(x_{1}(s), x_{2}(s)\right), s \in$ $[-\pi, \pi]$. Suppose that there exists a polynomial with complex coefficients

$$
p(w)=\sum_{k=0}^{N} a_{k} w^{k}, \quad w \in \mathbf{C}, N \in \mathbf{N},
$$

such that

$$
x_{1}(s)+i x_{2}(s)=p\left(e^{i s}\right), \quad s \in[-\pi, \pi] .
$$

Then $D$ has the Pompeiu property, unless $p(w)=a w+b$, for some $a \in$ $\mathbf{C} \backslash\{0\}, b \in \mathbf{C}$.

If $p(z)=a w+b,(1.3)$ represents a circle centered at $b$ with radius $|a|$. To provide some motivation for our result we discuss an example. The conchoid of the circle is the curve whose equation in polar coordinates is given by

$$
\rho(s)=a \cos s+b, \quad b \geq a>0, s \in[-\pi, \pi] .
$$

When $a=b$ the conchoid has a cusp at the origin of the type $x_{1}=-\left|x_{2}\right|^{2 / 3}$, whereas for $a<b$ the curve is real analytic. For $2 a \leq b$ the conchoid bounds 

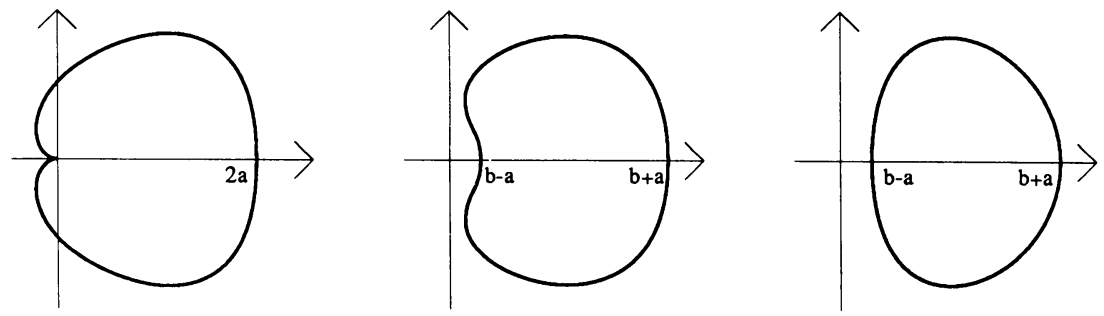

FIGURE 1

a convex region $D \subset \mathbf{R}^{2}$. It is an exercise to show that in this case if $m(D)$ and $M(D)$ are as in Theorem $\mathrm{B}$ above, then

$$
m(D)>\frac{1}{2} M(D)
$$

Figure 1 represents the three possibilities for (1.4).

Because of the above observations, the Pompeiu property for a domain $D \subset$ $\mathbf{R}^{2}$ bounded by a conchoid of the circle, cannot be deduced from the existing results. Theorem A does not apply to the case $a=b$, while (1.5) prevents the use of Theorem B in the case $2 a \leq b$. The intermediate case $a<b<2 a$ remains uncovered as well. Using the formula $\cos s=\left(e^{i s}+e^{-i s}\right) / 2$ we can rewrite (1.4) as follows

$$
\rho(s) e^{i s}=\frac{a}{2} e^{2 i s}+b e^{i s}+\frac{a}{2}=p\left(e^{i s}\right),
$$

where $p(w)=\frac{a}{2} w^{2}+b w+\frac{a}{2}$. From the theorem above we then conclude that every domain bounded by a conchoid has the Pompeiu property. The proof of the theorem relies on the following result of Brown, Schreiber, and Taylor [BST].

Theorem C. Let $D \subset \mathbf{R}^{2}$ be a bounded set. Then $D$ has the Pompeiu property iff for no $\alpha \in \mathbf{C} \backslash\{0\}$ does the complexified Fourier transform of the characteristic function of $D$,

$$
\hat{\chi}_{D}(\zeta)=\int_{D} e^{-i\langle\zeta, x\rangle} d x, \quad \zeta=\left(\zeta_{1}, \zeta_{2}\right) \in \mathbf{C}^{2}
$$

vanish identically on a set

$$
M_{\alpha}=\left\{\zeta=\left(\zeta_{1}, \zeta_{2}\right) \in \mathbf{C}^{2} \mid \zeta_{1}^{2}+\zeta_{2}^{2}=\alpha\right\}
$$

Since we are concerned with simply connected domains by virture of [W1, Theorem 1] we can assume $\alpha>0$. In $\S 3$ we obtain an asymptotic estimate of (1.6) when $D$ is a domain as in the theorem, $\zeta \in M_{\alpha}$, and $\zeta \rightarrow \infty$, see Theorem 3.1. In $\S 4$ we deduce the Pompeiu property for $D$ from this estimate and Theorem C. Our approach is based on Riemann's method of the steepest descent. We mention that the idea to attack the Pompeiu problem by using asymptotic expansions of $\hat{\chi}_{D}$ has been inspired to us by Berenstein's paper [B]. 
We would like to thank Giovanni Dore for so graciously helping us with the drawings at the Macintosh.

\section{Preliminary Reductions}

The integral (1.6), is, in general, difficult to deal with directly. The purpose of this section is to bring it to a form which better lends itself to an application of the method of the steepest descent. Since the Pompeiu property is invariant under translations we can, without loss of generality, assume that the polynomial $p(w)$ in the theorem is of the type

$$
p(w)=\sum_{k=M}^{N} a_{k} w^{k}, \quad w \in \mathbf{C},
$$

for a certain $1 \leq M<N$, where $a_{k} \in \mathbf{C}, k=M, \ldots, N, a_{M} \neq 0, a_{N} \neq 0$. If $x(s)=\left(x_{1}(s), x_{2}(s)\right)$ is as in the statement of the theorem, we obtain

$$
\hat{\chi}_{\partial D}(i \zeta)=\int_{-\pi}^{\pi} \exp (\langle x(s), \zeta\rangle)\left(x_{1}^{\prime}(s)+i x_{2}^{\prime}(s)\right) d s .
$$

We wish to study the asymptotic behavior of (2.2) when $\zeta=\left(\zeta_{1}, \zeta_{2}\right) \rightarrow \infty$ along the algebraic variety $M_{-\alpha}$ in $\mathbf{C}^{2}$. Since we are interested in establishing the Pompeiu property for the domain $D$ enclosed by (1.3), and the latter is invariant under dilation, translation, and rotation, we can, without loss of generality, make the following assumption on the polynomial $p$ in (2.1). $a_{M}$ can be chosen to be one. If we write $a_{N}=\alpha_{N}+i \beta_{N}$, we can choose $\beta_{N}=0$ and $\alpha_{N}<0$. In fact, letting $a_{N}=\rho e^{i \theta}$, if $\psi \in[0,2 \pi]$ is to be chosen, we have

$$
e^{i \psi} p\left(e^{i s}\right)=e^{i(M s+\psi)}+\cdots+\rho e^{i(N s+\theta+\psi)}, \quad s \in[-\pi, \pi] .
$$

At this point we choose $\psi=M(\theta+\pi) /(N-M)$ and perform the change of variable $\tau=s+\psi$. In conclusion, we can assume that $p\left(e^{i s}\right)$ takes the form

$$
p\left(e^{i s}\right)=e^{i M s}+a_{M+1} e^{i(M+1) s}+\cdots+a_{N} e^{i N s}, \quad \text { with } a_{N}<0 .
$$

Now we let $\zeta=\left(\zeta_{1}, \zeta_{2}\right) \in M_{-\alpha}, \alpha>0$, and we choose $\zeta_{1}=r, \zeta_{2}=i t$, with $r>0, t<0$. The condition that $\zeta \in M_{-\alpha}$ is then

$$
\zeta_{1}^{2}+\zeta_{2}^{2}=r^{2}-t^{2}=-\alpha
$$

This yields for $r \rightarrow+\infty$

$$
t-r=\frac{\alpha}{2 r}\left(1+O\left(\frac{1}{r^{2}}\right)\right) .
$$

Recalling (1.3), setting $q(s)=p\left(e^{i s}\right), s \in[-\pi, \pi]$, we rewrite (2.2) as follows:

$$
\hat{\chi}_{\partial D}(i \zeta)=\int_{-\pi}^{\pi} \exp \left[r q(s)+i(t-r) x_{2}(s)\right] q^{\prime}(s) d s .
$$


At this point we shift the integration path in (2.5) to the line $s+i \varepsilon \ln r, s \in$ $[-\pi, \pi]$, where

$$
\varepsilon=\frac{2}{M+N}
$$

We note that

$$
q(s+i \varepsilon \ln r)=\sum_{k=M}^{N} a_{k} e^{i k s} r^{-\varepsilon k}
$$

Moreover, letting $a_{k}=\alpha_{k}+i \beta_{k}, k=M, \ldots, N$, we have

$$
\begin{aligned}
x_{2}(s+i \varepsilon \ln r)= & \frac{1}{2} \sum_{k=M}^{N}\left(\frac{\alpha_{k}}{i}+\beta_{k}\right) e^{i k s} r^{-\varepsilon k} \\
& +\frac{1}{2} \sum_{k=M}^{N}\left(\beta_{k}-\frac{\alpha_{k}}{i}\right) e^{-i k s} r^{\varepsilon k} .
\end{aligned}
$$

Equations (2.4) and (2.8) yield

$$
i(t-r) x_{2}(s+i \varepsilon \ln r)=\sum_{k=M}^{N} \frac{\alpha}{4}\left(\beta_{k}-\frac{\alpha_{k}}{i}\right) e^{-i k s} r^{\varepsilon k-1}+O\left(r^{-(\varepsilon M+1)}\right),
$$

where we have denoted by $O\left(r^{-(\varepsilon M+1)}\right)$ a function whose absolute value is bounded uniformly for $r$ large and $s \in[-\pi, \pi]$ by a constant depending only on the polynomial $p$ in (2.1). Putting (2.7) and (2.9) together we obtain

$$
\begin{aligned}
r q(s+ & i \varepsilon \ln r)+i(t-r) x_{2}(s+i \varepsilon \ln r) \\
= & r^{-\varepsilon M+1}\left(e^{i M s}-\frac{\alpha a_{N}}{4} e^{-i N s}\right)+r^{-\varepsilon(M+1)+1} \sum_{k=M+1}^{N} a_{k} e^{i k s} r^{-\varepsilon(k-M-1)} \\
& +r^{\varepsilon(N-1)-1} \sum_{k=M+1}^{N-1} \frac{i \alpha}{4}\left(\beta_{k}-\frac{\alpha_{k}}{i}\right) e^{-i k s} r^{-\varepsilon(N-k-1)}+O\left(r^{-(\varepsilon M+1)}\right) .
\end{aligned}
$$

A simple calculation now gives

$$
q^{\prime}(s+i \varepsilon \ln r)=i r^{-\varepsilon M} \sum_{k=M}^{N} k a_{k} e^{i k s} r^{-\varepsilon(k-M)} .
$$

At this point we introduce some notation. For $z \in \mathbf{C}$ we set

$$
\varphi(z)=e^{i M z}-\frac{\alpha a_{N}}{4} e^{-i N z},
$$

$$
\begin{aligned}
\dot{\psi}(z ; r)= & \sum_{h=0}^{N-M-1}\left[a_{h+M+1} e^{i(h+M+1) z}\right. \\
& \left.+\frac{i \alpha}{4}\left(\beta_{N-h-1}-\frac{\alpha_{N-h-1}}{i}\right) e^{-i(N-h-1) z}\right] r^{-\varepsilon h},
\end{aligned}
$$




$$
g(z ; r)=\sum_{h=0}^{N-M}(h+M) a_{h+M} e^{i(h+M) z} r^{-\varepsilon h} ;
$$

By Cauchy's theorem and the periodicity of the integrand in (2.5) we deduce from $(2.10)-(2.14)$

$$
\begin{aligned}
\hat{\chi}_{\partial D}(i \zeta)=i r^{-\varepsilon M} \int_{-\pi}^{\pi} \exp \left[r^{-\varepsilon M+1} \varphi(s)\right. & \\
& \left.\quad+r^{-\varepsilon(M+1)+1} \psi(s ; r)+O\left(r^{-(\varepsilon M+1)}\right)\right] \cdot g(s ; r) d s .
\end{aligned}
$$

Since as $r \rightarrow+\infty, \exp \left[O\left(r^{-(\varepsilon M+1)}\right)\right]=1+O\left(r^{-(\varepsilon M+1)}\right)$, setting

$$
k(s ; r)=g(s ; r)\left(1+O\left(r^{-(\varepsilon M+1)}\right)\right),
$$

we finally obtain from $(2.15)$

$$
\hat{\chi}_{\partial D}(i \zeta)=i r^{-\varepsilon M} \int_{-\pi}^{\pi} \exp \left[r^{-\varepsilon M+1}\left(\varphi(s)+r^{-\varepsilon} \psi(s ; r)\right)\right] k(s ; r) d s .
$$

We remark that $\psi(s ; r)$ is a polynomial in $r^{-\varepsilon}$ whose coefficients are trigonometrical polynomials in $s \in[-\pi, \pi]$.

\section{ASYMPTOTIC ANALYSIS OF THE INTEGRAL (2.17)}

The work done in the previous section will enable us to carry through the asymptotic analysis of (2.2). The study of the Fourier integral (2.17) can be attacked by the method of the steepest descent. The phase in (2.17) is the sum of the function $\varphi$ to which we can apply the method, see Lemma 3.1 below, plus a perturbation that for $r$ large moves the critical points of $\varphi$ by little.

Lemma 3.1. Let $M, N$ be fixed as in (2.1) and let $\varphi$ be defined by (2.12) (recall that $\left.a_{N}<0\right)$. Then, the point

$$
z_{0}=\frac{1}{i} \ln \left(\frac{\alpha\left|a_{N}\right| N}{4 M}\right)^{1 /(N+M)}
$$

is a simple critical point of $\varphi$, i.e., $\varphi^{\prime}\left(z_{0}\right)=0$ and $\varphi^{\prime \prime}\left(z_{0}\right) \neq 0$. Moreover, there exist $\eta \in \mathbf{R}$ and a $C^{1}$ path $\gamma$ contained in the region

$$
\operatorname{Re} \varphi(z)<\operatorname{Re} \varphi\left(z_{0}\right),
$$

with the exception of the paint $z=z_{0}$, which joins the point $(-\pi, \eta)$ to the point $(\pi, \eta)$.

Proof. It is immediate to check that for $z_{0}$ as in (3.1) we have $\varphi^{\prime}\left(z_{0}\right)=0$, $\varphi^{\prime \prime}\left(z_{0}\right) \neq 0$, and that $\operatorname{Re} \varphi\left(z_{0}\right)>0$. The easy, although tedious, details of the second part of the lemma are left to the reader. The latter can easily convince himself that, qualitatively, the region $\operatorname{Re} \varphi(z)<\operatorname{Re} \varphi\left(z_{0}\right)$ looks like that earmarked by $a+$ in Figure 2 . 


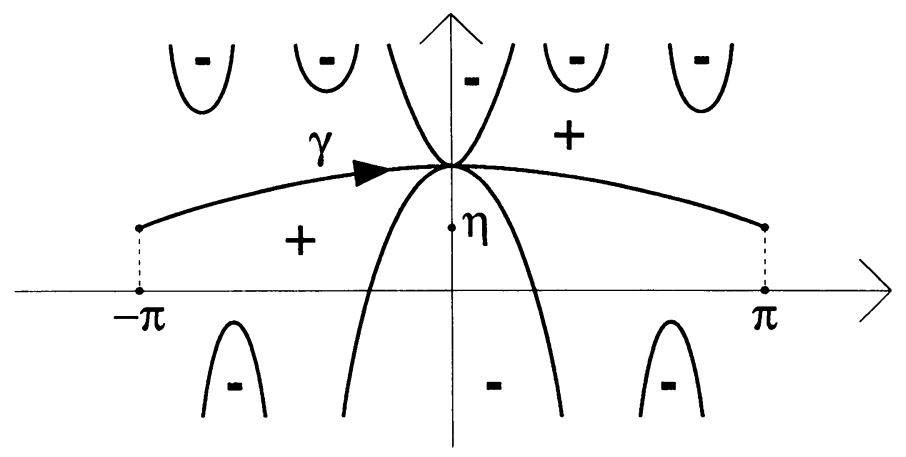

FIGURE 2

We now let $\tilde{\varphi}(z)=\varphi(z)-\varphi\left(z_{0}\right)$ and define

$$
\begin{aligned}
\Phi(z ; r) & =\varphi(z)-\varphi\left(z_{0}\right)+r^{-\varepsilon} \psi(z ; r) \\
& =\tilde{\varphi}(z)+r^{-\varepsilon} \psi(z ; r) .
\end{aligned}
$$

We observe that

$$
\tilde{\varphi}\left(z_{0}\right)=0, \quad \tilde{\varphi}^{\prime}\left(z_{0}\right)=0, \quad \tilde{\varphi}^{\prime \prime}\left(z_{0}\right) \neq 0 .
$$

Making the substitution $\sigma=r^{-\varepsilon}$ (3.2) becomes

$$
\tilde{\Phi}(z ; \sigma)=\tilde{\varphi}(z)+\sigma \tilde{\psi}(z ; \sigma),
$$

where we have set $\tilde{\Phi}(z ; \sigma)=\Phi\left(z ; \sigma^{-1 / \varepsilon}\right), \tilde{\psi}(z ; \sigma)=\psi\left(z ; \sigma^{-1 / \varepsilon}\right)$. Using (3.3) we infer

$$
\tilde{\Phi}\left(z_{0} ; 0\right)=0, \quad \frac{\partial \tilde{\Phi}}{\partial z}\left(z_{0} ; 0\right)=0, \quad \frac{\partial^{2} \tilde{\Phi}}{\partial z^{2}}\left(z_{0} ; 0\right) \neq 0 .
$$

By (3.5) and Dini's complex implicit function theorem, we can then write

$$
\tilde{\Phi}(z ; 0)=f(\sigma)+(z-z(\sigma))^{2} E(z ; \sigma),
$$

with $f(\sigma), z(\sigma)$ analytic functions of $\sigma$ in a neighborhood of $\sigma=0, z(0)=$ $z_{0}$, and $E(z ; 0)$ analytic function of $(z ; \sigma)$ in a neighborhood of $\left(z_{0}, 0\right)$ with

$$
E\left(z_{0} ; 0\right) \neq 0 \text {. }
$$

We remark that $f(0)=\tilde{\Phi}\left(z_{0} ; 0\right)=0$. Next, we observe that, because of (3.4), for any compact set $K \subset\left\{z \mid \operatorname{Re} \varphi(z)<\operatorname{Re} \varphi\left(z_{0}\right)\right\}$ there exists a $\delta=$ $\delta_{K}>0$ such that $K \subset\{z \mid \operatorname{Re} \tilde{\Phi}(z ; \sigma)<\operatorname{Re} \tilde{\Phi}(z(\sigma) ; \sigma)\}$ for any $0<\sigma<\delta$. Let $\gamma$ be the path whose existence is claimed in Lemma 3.1, and let $\rho>$ 0 be fixed sufficiently small. Then there exists $\delta=\delta(\rho)>0$ such that for $\sigma \leq \delta, \gamma \backslash[\gamma \cap\{z|| z-z(\sigma) \mid<\rho\}]$ is contained in a compact subset $H$ of $\left\{z \mid \operatorname{Re} \varphi(z)<\operatorname{Re} \varphi\left(z_{0}\right)\right\}$. By the previous remark, for $\sigma$ sufficiently small $H$ is 
also contained in $\{z \mid \operatorname{Re} \tilde{\Phi}(z ; \sigma)<\operatorname{Re} \tilde{\Phi}(z(\sigma) ; \sigma)\}$. Therefore, we can deform the path $\gamma$ into a new path $\gamma_{\sigma}$ such that for every sufficiently small $\sigma$

$\gamma_{\sigma}$ passes through $z(\sigma)$,

$$
\begin{gathered}
\gamma_{\sigma} \backslash\{z(\sigma)\} \subset\{\operatorname{Re} \tilde{\Phi}(z ; \sigma)<\operatorname{Re} \tilde{\Phi}(z(\sigma) ; \sigma)\}, \\
\gamma_{\sigma} \equiv \gamma \text { outside }\{z|| z-z(\sigma) \mid<\rho\} .
\end{gathered}
$$

See Figure 3.

By Cauchy's theorem and (2.17) we obtain

$$
\begin{aligned}
& \hat{\chi}_{\partial D}(i \zeta)=i r^{-\varepsilon M} \exp \left[r^{-\varepsilon M+1} \varphi\left(z_{0}\right)\right] \\
& \cdot \int_{-\pi}^{\pi} \exp \left[r^{-\varepsilon M+1}\left(\tilde{\varphi}(s)+r^{-\varepsilon} \psi(s ; r)\right)\right] k(s ; r) d s \\
& =i r^{-\varepsilon M} \exp \left[r^{-\varepsilon M+1} \varphi\left(z_{0}\right)\right] \\
& \cdot \int_{\pi} \exp \left[r^{-\varepsilon M+1}\left(\tilde{\varphi}(z)+r^{-\varepsilon} \psi(z ; r)\right)\right] k(z ; r) d z \\
& =i r^{-\varepsilon M} \exp \left[r^{-\varepsilon M+1} \varphi\left(z_{0}\right)\right]\{\mathrm{I}(r)+\mathrm{II}(r)\},
\end{aligned}
$$

where we have set (cf. (3.2))

$$
\begin{aligned}
& \mathrm{I}(r)=\int_{\gamma_{\sigma} \cap\{z|| z-z(\sigma) \mid \leq \rho\}} \exp \left[r^{-\varepsilon M+1} \Phi(z ; r)\right] k(z ; r) d z, \\
& \mathrm{II}(r)=\int_{\gamma_{\sigma} \cap\{z|| z-z(\sigma) \mid>\rho\}} \exp \left[r^{-\varepsilon M+1} \Phi(z ; r)\right] k(z ; r) d z .
\end{aligned}
$$

We first analyze $\mathrm{II}(r)$. We note that by (3.10)

$$
\mathrm{II}(r)=\int_{\gamma \cap\{z|| z-z(\sigma) \mid>\rho\}} \exp \left[r^{-\varepsilon M+1} \Phi(z ; r)\right] k(z ; r) d z .
$$

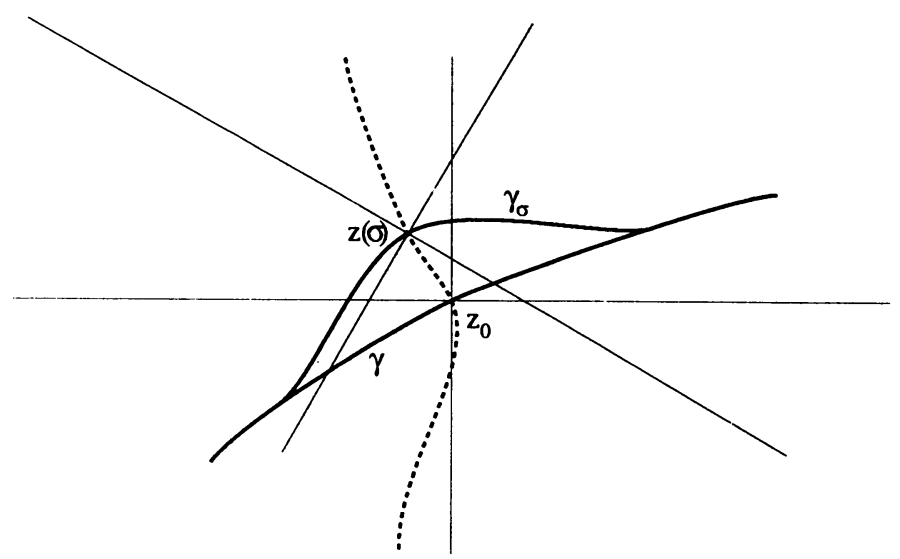

FIGURE 3 
For $\sigma$ sufficiently small we have

$$
[\gamma \cap\{z|| z-z(\sigma) \mid>\rho\}] \subset\left[\gamma \cap\left\{z|z-z(\sigma)| \geq \frac{\rho}{2}\right\}\right],
$$

and the latter is a compact subset of $\{z \mid \operatorname{Re} \tilde{\varphi}(z)<0\}$, recall (3.3). Therefore, for a suitable $\tau>0, \operatorname{Re} \tilde{\varphi}(z)<-\tau$ on $\gamma \cap\left\{z|| z-z_{0} \mid \geq \frac{\rho}{2}\right\}$. It follows that for $\sigma$ sufficiently small

$$
\begin{aligned}
\operatorname{Re} \tilde{\Phi}(z ; \sigma) & =\operatorname{Re} \tilde{\varphi}(z)+\sigma \operatorname{Re} \tilde{\psi}(z ; \sigma) \\
& \leq-\tau+\sigma \operatorname{Re} \tilde{\psi}(z ; \sigma) \leq-\tau / 2
\end{aligned}
$$

Equation (3.12) allows to conclude that for a $C=C(\tau)>0$

$$
\mathrm{II}(r)=O\left(\exp -\left[C r^{-\varepsilon M+1}\right]\right) \quad \text { as } r \rightarrow+\infty .
$$

We now examine $\mathrm{I}(r)$. (3.6) plays a crucial role. By it, recalling that $\sigma=r^{-\varepsilon}$, we can write for sufficiently small $\sigma$

$$
\begin{aligned}
\mathrm{I}\left(\sigma^{-1 / \varepsilon}\right)= & \int_{\gamma_{\sigma} \cap\{z|| z-z(\sigma) \mid<\rho\}} \exp \left[\sigma^{M-1 / \varepsilon} \tilde{\Phi}(z ; \sigma)\right] k\left(z ; \sigma^{-1 / \varepsilon}\right) d z \\
= & \int_{\gamma_{\sigma} \cap\{z|| z-z(\sigma) \mid<\rho\}} \exp \left[\sigma^{M-1 / \varepsilon}\left(f(\sigma)+(z-z(\sigma))^{2} E(z ; \sigma)\right)\right] k\left(z ; \sigma^{-1 / \varepsilon}\right) d z \\
= & \exp \left[\sigma^{M-1 / \varepsilon} f(\sigma)\right] \\
& \cdot \int_{\gamma_{\sigma} \cap\{z|| z-z(\sigma) \mid<\rho\}} \exp \left[\sigma^{M-1 / \varepsilon}(z-z(\sigma))^{2} E(z ; \sigma)\right] k\left(z ; \sigma^{-1 / \varepsilon}\right) d z
\end{aligned}
$$

If we set $\Psi(z ; \sigma)=(z-z(\sigma))^{2} E(z ; \sigma)$, then by (3.9) we have

$$
\begin{aligned}
& \{z \mid \operatorname{Re} \tilde{\Phi}(z ; \sigma)>\operatorname{Re} \tilde{\Phi}(z(\sigma) ; \sigma)\} \\
& \quad=\{z \mid \operatorname{Re} \tilde{\Psi}(z ; \sigma)>\operatorname{Re} \tilde{\Psi}(z(\sigma) ; \sigma)=0\} .
\end{aligned}
$$

Since $E\left(z_{0} ; 0\right) \neq 0$ we may write

$$
\Psi(z ; \sigma)=\{(z-z(\sigma))[A(z ; \sigma)+i B(z ; \sigma)]\}^{2}
$$

provided that $\left|z-z_{0}\right|$ and $\sigma$ are sufficiently small. We remark that our choice of the complex square root of a number $w$ is $\sqrt{w}=|w|^{1 / 2} \exp \left(i \frac{\arg w}{2}\right), 0<$ $\arg w<2 \pi$. Moreover, we may assume without loss of generality that

$$
B(z ; \sigma) \neq 0 \text { in a neighborhood of }\left(z_{0}, 0\right) \text {. }
$$

We write $z_{0}=x_{0}+i y_{0}, z=x+i y$ and $z(\sigma)=x(\sigma)+i y(\sigma)$.

Then (3.16) becomes

$$
\begin{aligned}
\Psi(z ; \sigma)=\{[(x-x(\sigma)) A(x, y ; \sigma)-(y-y(\sigma)) B(x, y ; \sigma)] \\
+i[(y-y(\sigma)) A(x, y ; \sigma)+(x-x(\sigma)) B(x, y ; \sigma)]\}^{2},
\end{aligned}
$$

where by abuse of notation we have denoted by $A(x, y ; \sigma)$ and $B(x, y ; \sigma)$ respectively the functions $A(x+i y ; \sigma)$ and $B(x+i y ; \sigma)$. We define

$$
F(x, y ; \sigma)=(x-x(\sigma)) A(x, y ; \sigma)-(y-y(\sigma)) B(x, y ; \sigma) .
$$


We want to determine a one-parameter family of curves $x \mapsto y(x ; \sigma)$ such that

$$
\left\{\begin{array}{l}
F(x, y(x, \sigma) ; \sigma)=0 \\
y(x(\sigma) ; \sigma)=y(\sigma)
\end{array}\right.
$$

By Dini's theorem this is possible for $x$ close to $x_{0}$ since by (3.17) we have

$$
\left.\frac{\partial F}{\partial y}\right|_{\substack{x=x(\sigma) \\ y=y(\sigma)}}=-B(x(\sigma), y(\sigma) ; \sigma) \neq 0,
$$

for $\sigma$ small, and, moreover, we have $F(x(\sigma), y(\sigma) ; \sigma)=0$. In conclusion, we have by (3.21) and (3.19)

$$
\begin{aligned}
\left.\Psi\right|_{y=y(x ; \sigma)} & =-[(y(x ; \sigma)-y(\sigma)) A(x, y(x ; \sigma) ; \sigma) \\
& +(x-x(\sigma)) B(x, y(x ; \sigma) ; \sigma)]^{2} \\
& \stackrel{\text { def }}{=}-M(x ; \sigma)^{2} .
\end{aligned}
$$

Now we want to show that

$$
M(x ; \sigma)=(x-x(\sigma)) L(x ; \sigma)
$$

with $L(x ; \sigma) \neq 0$ in a neighborhood of $\left(x_{0}, 0\right)$. We observe that because of (3.20),

$$
M(x(\sigma) ; \sigma)=0 .
$$

Therefore, for $x$ close to $x_{0}$ and $\sigma$ small we have

$$
M(x ; \sigma)=\frac{\partial M}{\partial x}(x(\sigma) ; \sigma)(x-x(\sigma))+O\left((x-x(\sigma))^{2}\right)
$$

(3.21) yields

$$
\frac{\partial M}{\partial x}(x(\sigma) ; \sigma)=B(x(\sigma), y(\sigma) ; \sigma)+\frac{\partial y}{\partial x}(x(\sigma) ; \sigma) A(x(\sigma), y(\sigma) ; \sigma) .
$$

Moreover, from (3.20) we obtain

$$
\frac{\partial y}{\partial x}(x(\sigma) ; \sigma)=-\frac{\frac{\partial F}{\partial x}(x(\sigma), y(\sigma) ; \sigma)}{\frac{\partial F}{\partial y}(x(\sigma), y(\sigma) ; \sigma)}=\frac{A(x(\sigma), y(\sigma) ; \sigma)}{B(x(\sigma), y(\sigma) ; \sigma)}
$$

In (3.25) we have used (3.19). Inserting (3.25) into (3.24), using (3.16) and (3.17), we finally have for $\sigma$ small enough

$$
\frac{\partial M}{\partial x}(x(\sigma) ; \sigma)=\frac{A(x(\sigma), y(\sigma) ; \sigma)^{2}+B(x(\sigma), y(\sigma) ; \sigma)^{2}}{B(x(\sigma), y(\sigma) ; \sigma)} \neq 0 .
$$

Plugging this information in (3.23) yields (3.22). We conclude that along the curve $x \mapsto y(x ; \sigma)$, and locally around the point $\left(x_{0}, 0\right)$, we have $\operatorname{Re} \Psi>0$, except at the point $x=x(\sigma)$ where $\operatorname{Re} \Psi=0$. Therefore, there exists a $\delta>0$ such that for $\sigma \leq \delta,|z-z(\sigma)| \leq \delta$ and $x \neq x(\sigma)$, the curve $x \mapsto y(x ; \sigma)$ 


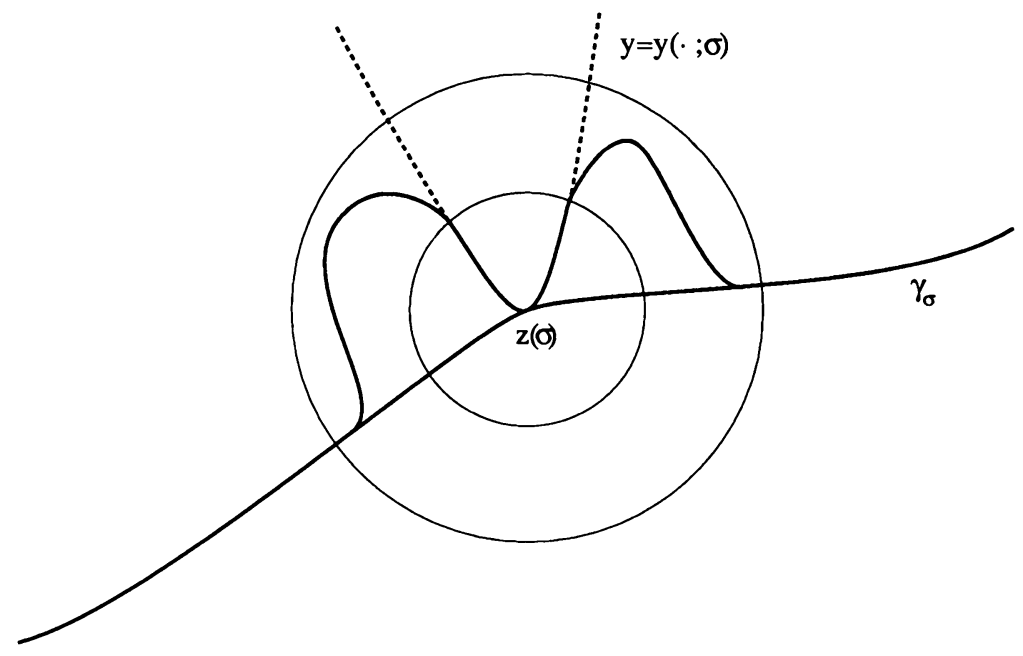

FIGURE 4

is contained in the region $\{z \mid \operatorname{Re} \Psi(z ; \sigma)>\operatorname{Re} \Psi(z(\sigma) ; \sigma)=0\}$, see (3.15). Using this observation we can deform the path $\gamma_{\sigma}$ into a new path, $\tilde{\gamma}_{\sigma}$, which coincides with $\gamma_{\sigma}$ for $|z-z(\sigma)| \geq 2 \delta$, with $y=y(\cdot ; \sigma)$ for $|z-z(\sigma)| \leq \delta$, and which for $\delta \leq|z-z(\sigma)| \leq 2 \delta$ is a continuous path that joins the two branches without exiting the region (3.15) (see Figure 4).

We now return to (3.14). We choose $\rho=2 \delta$ in (3.14). Another application of Cauchy's theorem gives

$$
\begin{aligned}
\int_{\gamma_{\sigma} \cap\{z|| z-z(\sigma) \mid<2 \delta\}} \exp \left[\sigma^{M-1 / \varepsilon}(z-z(\sigma))^{2} E(z ; \sigma)\right] k\left(z ; \sigma^{-1 / \varepsilon}\right) d z \\
=\left(\int_{\tilde{\gamma}_{\sigma} \cap\{z|| z-z(\sigma) \mid<\delta\}}+\int_{\tilde{\gamma}_{\sigma} \cap\{z|\delta \leq| z-z(\sigma) \mid \leq 2 \delta\}}\right) \\
\quad \cdot \exp \left[\sigma^{M-1 / \varepsilon}(z-z(\sigma))^{2} E(z ; \sigma)\right] k\left(z ; \sigma^{-1 / \varepsilon}\right) d z \\
=I^{\prime}(\sigma)+I^{\prime \prime}(\sigma) .
\end{aligned}
$$

There exists $\tau=\tau(\delta)>0$ for which that part of $\tilde{\gamma}_{\sigma}$ contained in the annulus $\{z|\delta \leq| z-z(\sigma) \mid \leq 2 \delta\}$ lies in the region $\{z \mid \operatorname{Re} \Psi(z ; \sigma)>0\}$. Hence, as for (3.13) we can conclude that as $\sigma \rightarrow 0$

$$
I^{\prime \prime}(\sigma)=O\left(\sigma^{k}\right) \quad \text { for every } k \in \mathbf{N}
$$

We are left with estimating $\mathrm{I}^{\prime}(\sigma)$. Recalling that $(z-z(\sigma))^{2} E(z ; \sigma)=\Psi(z ; \sigma)$, 
and using (3.21), (3.22), we have

$$
\begin{aligned}
\int_{\tilde{\gamma}_{\sigma} \cap\{z|| z-z(\sigma) \mid<\delta\}} \exp \left[\sigma^{M-1 / \varepsilon}(z-z(\sigma))^{2} E(z ; \sigma)\right] k\left(z ; \sigma^{-1 / \varepsilon}\right) d z \\
=\int_{\tilde{\gamma}_{\sigma} \cap\{z|| z-z(\sigma) \mid<\delta\}} \exp \left[-\sigma^{M-1 / \varepsilon}(x-x(\sigma))^{2} L(x ; \sigma)^{2}\right] k\left(z ; \sigma^{-1 / \varepsilon}\right) d z \\
=\int_{x(\sigma)-\alpha(\sigma)}^{x(\sigma)+\beta(\sigma)} \exp \left[-\sigma^{M-1 / \varepsilon}(x-x(\sigma))^{2} L(x ; \sigma)^{2}\right] \\
\cdot k\left(x+i y(x ; \sigma) ; \sigma^{-1 / \varepsilon}\right)\left[1+i \frac{\partial y}{\partial x}(x ; \sigma)\right] d x,
\end{aligned}
$$

where for $\sigma \leq \delta, \alpha(\sigma), \beta(\sigma)$ are suitable numbers with

$$
\alpha(\sigma) \geq \alpha_{0}>0, \quad \beta(\sigma) \geq \beta_{0}>0, \quad \text { for } \sigma \leq \delta .
$$

At this point we perform the change of variable

$$
u=(x-x(\sigma)) L(x ; \sigma)
$$

in the last integral in (3.28). Since by (3.22) $L(x ; \sigma) \neq 0$ in a neighborhood of $\left(x_{0}, 0\right)$, we can write

$$
x=x(\sigma)+u q(u ; \sigma),
$$

with $q(u ; \sigma) \neq 0$ in a neighborhood of $(0,0)$. Using (3.29), (3.28) becomes

$$
\int_{-\infty}^{+\infty} \exp \left[-\sigma^{M-1 / \varepsilon} u^{2}\right] \Omega(u ; \sigma) d u+O\left(\sigma^{k}\right)
$$

for every $k \in \mathbf{N}$, where in virtue of (3.30) we have set

$$
\begin{aligned}
\Omega(u ; \sigma)= & \chi(u) k\left(x(\sigma)+u q(u ; \sigma)+i y(x(\sigma)+u q(u ; \sigma)) ; \sigma^{-1 / \varepsilon}\right) \\
& \cdot\left[1+i \frac{\partial y}{\partial x}(x(\sigma)+u q(u ; \sigma) ; \sigma)\right]\left(q(u ; \sigma)+u \frac{\partial q}{\partial u}(u ; \sigma)\right) .
\end{aligned}
$$

In the definition of $\Omega(u ; \sigma), \chi(u)$ is a cut-off function, $\chi \in C^{\infty}(\mathbf{R}), \chi \equiv 1$ on a neighborhood of $u=0$. We write

$$
\Omega(u ; \sigma)=\Omega(0 ; \sigma)+u R(u ; \sigma)
$$

with $R$ a bounded function on $\mathbf{R} \times[0, \delta]$. By performing the change of variable $\sigma^{(M-1 / \varepsilon) / 2} u=v$ in the integral in (3.31), we obtain

$$
\begin{aligned}
\int_{-\infty}^{+\infty} \exp \left[-\sigma^{M-1 / \varepsilon}\right. & \left.u^{2}\right] \Omega(u ; \sigma) d u \\
=\sigma^{-(M-1 / \varepsilon) / 2} & \{\sqrt{\pi} \Omega(0 ; \sigma) \\
& \left.\quad+\sigma^{-(M-1 / \varepsilon) / 2} \int_{-\infty}^{+\infty} \exp \left[-v^{2} R\left(\sigma^{-(M-1 / \varepsilon) / 2} v ; \sigma\right)\right] d v\right\} .
\end{aligned}
$$


(3.25) gives

$$
\Omega(0 ; \sigma)=k\left(z(\sigma) ; \sigma^{-1 / \varepsilon}\right) \frac{A(x(\sigma), y(\sigma) ; \sigma)-i B(x(\sigma), y(\sigma) ; \sigma)}{A^{2}(x(\sigma), y(\sigma) ; \sigma)+B^{2}(x(\sigma), y(\sigma) ; \sigma)} .
$$

Recalling now (2.14), (2.16), we rewrite (3.33) as follows

$$
\Omega(0 ; \sigma)=\frac{i M \exp (i M z(\sigma))}{A(x(\sigma), y(\sigma) ; \sigma)-i B(x(\sigma), y(\sigma) ; \sigma)}+O(\sigma) \text {. }
$$

From (3.32) and (3.33) we then obtain

$$
\mathrm{I}^{\prime}(\sigma)=\sigma^{-(M-1 / \varepsilon) / 2}\left\{\frac{i \sqrt{\pi} M \exp (i M z(\sigma))}{A(x(\sigma), y(\sigma) ; \sigma)-i B(x(\sigma), y(\sigma) ; \sigma)}+o(1)\right\} .
$$

Equations (3.6) and (3.16) imply

$$
A\left(x_{0}, y_{0} ; 0\right)+i B\left(x_{0}, y_{0} ; 0\right)=\sqrt{\frac{\varphi^{\prime \prime}\left(z_{0}\right)}{2}}=i\left|\frac{\varphi^{\prime \prime}\left(z_{0}\right)}{2}\right|^{1 / 2} \text {. }
$$

Finally, recalling (2.6), from (3.11), (3.13), (3.26), (3.27), (3.34), and (3.35), we conclude with the following

Theorem 3.1. Let $D \subset \mathbf{R}^{2}$ be a bounded, simply connected open set whose boundary, $\partial D$, is parametrized by $x_{1}(s)+i x_{2}(s)=p(s)=\sum_{k=M}^{N} a_{k} e^{i k s}, s \in[-\pi, \pi]$, with $N, M \in \mathbf{N}, 1 \leq M<N, a_{k} \in \mathbf{C}, k=M, \ldots, N, a_{M} \neq 0, a_{N} \neq 0$. Then, if $\zeta \in \mathbf{C}^{2}, \zeta=(r, i t), r, t>0, t^{2}-r^{2}=\alpha, \alpha>0$, we have the following asymptotic expansion

$$
\hat{\chi}_{\partial D}\left(i\left|a_{M}\right| A_{\omega} \zeta\right)=\frac{e^{i \omega}}{\left|a_{M}\right|} r^{-(N-M) / 2(N+M)} G(r) \exp \left[r^{(N-M) /(N+M)}\left(\varphi\left(z_{0}\right)+\mu(r)\right)\right],
$$

where $z_{0}$ is as in (3.1), $\omega=M\left(\arg a_{N}+\pi\right) /(N-M)-\arg a_{M}$,

$$
A_{\omega}=\left(\begin{array}{cc}
\cos \omega & \sin \omega \\
-\sin \omega & \cos \omega
\end{array}\right),
$$

$\varphi$ is defined by (2.12), and $G$ and $\mu$ are two functions such that

$$
G(r) \rightarrow \frac{\sqrt{2} M e^{i M z_{0}}}{i\left|\varphi^{\prime \prime}\left(z_{0}\right)\right|^{1 / 2}}, \quad \mu(r) \rightarrow 0 \quad \text { as } r \rightarrow+\infty
$$

\section{THE POMPEIU PROBLEM}

In this section we prove the theorem. The hard work has already been done in proving Theorem 3.1. In fact, the proof of the theorem is, at this point, a straightforward consequence of the expansion (3.36) and of the quoted Theorem $\mathrm{C}$ of Brown, Schreiber, and Taylor. Let $\zeta=\left(\zeta_{1}+\zeta_{2}\right) \in \mathbf{C}^{2}$. Since by the divergence theorem

$$
\hat{\chi}_{\partial D}(\zeta)=\left(\zeta_{1}+\zeta_{2}\right) \hat{\chi}_{D}(\zeta)
$$


it is clear from (3.36) that for no $\alpha>0$ can $\hat{\chi}_{D}$ vanish identically on a set $M_{\alpha}$, see (1.7).

Remark. In the statements of the theorem and of Theorem 3.1 we have assumed that the degree $N$ of the polynomial $p$ is strictly larger than one. If $M=N=$ 1 , then $\varepsilon=2 /(N+M)=1$ in (2.6), and (2.17) becomes

$$
\hat{\chi}_{\partial D}(i \zeta)=i r^{-1} \int_{-\pi}^{\pi} \exp [\varphi(s)] k(s ; r) d s,
$$

where now

$$
\varphi(s)=e^{i s}-\frac{\alpha}{4} e^{-i s} \text { and } k(s ; r)=e^{i s}\left(1+O\left(r^{-2}\right)\right) .
$$

Then (4.1) gives as $r \rightarrow+\infty$

$$
\hat{\chi}_{\partial D}(i s)=i r^{-1} \int_{-\pi}^{\pi} \exp [\varphi(s)] e^{i s} d s+O\left(r^{-\varepsilon}\right) .
$$

Now, a computation yields

$$
\hat{\chi}_{\partial D}(i s)=-i \frac{\alpha}{2} \frac{1}{r} J_{1}(\sqrt{-\alpha})+O\left(r^{-\varepsilon}\right),
$$

where $J_{1}$ is the Bessel function of the first kind and order one. We choose $\alpha<0$ such that $\sqrt{-\alpha}$ is a zero of $J_{1}$. Then (4.2) yields no information. Rightly so, however, since we know that the circle does not have the Pompeiu property.

\section{BIBLIOGRAPHY}

[B] C. A. Berenstein, An inverse spectral theorem and its relation to the Pompeiu problem, J. Analyse Math. 37 (1980), 128-144.

[BK] L. Brown and J. P. Kahane, A note on the Pompeiu problem for convex domains, Math. Ann. 259 (1982), 107-110.

[BST] L. Brown, B. M. Schreiber and A. B. Taylor, Spectral synthesis and the Pompeiu problem, Ann. Inst. Fourier (Grenoble) 23 (1973), 125-154.

[C] L. A. Caffarelli, The regularity of free boundaries in higher dimensions, Acta Math. 139 (1977), 155-184.

[Ch] L. Chakalov, Sur un problème de D. Pompeiu, Ann. Univ. Sofia Fac. Phys. Math. 40 (1944), $1-44$.

[P1] D. Pompeiu, Sur certains systèmes d'équations linéaires et sur une propriété intégrale des fonctions de plusieurs variables, C. R. Acad. Sci. Paris 188 (1929), 1.138-1.139.

[P2] _ Sur une propriété intégrales des fonctions de deux variables réelles, Bull. Sci. Acad. Royale Belgique 15 (1929), 265-269.

[R] B. Riemann, Sullo svolgimento del quoziente di due serie ipergeometriche in funzione continua infinita, Complete Works, Dover, New York, 1953.

[W1] S. A. Williams, A partial solution of the Pompeiu problem, Math. Ann. 223 (1976), 183-190.

[W2] _ Analyticity of the boundary for Lipschitz domains without the Pompeiu property, Indiana Univ. Math. J. 30 (1981), 357-369.

Department of Mathematics, Northwestern University, Evanston, IllinoIs 60208

Dipartimento di Matematica, Universita di Bologna, 40126 Bologna, Italy (Current address of Fausto Segala)

Current address (Nicola Garofalo): Department of Mathematics, Purdue University, West Lafayette, Indiana 47907 